\title{
Quantum Noether's Theorem and Conformal Field Theory: A Study of Some Models
}

\author{
Sebastiano Carpi \\ Dipartimento di Matematica, Università di Roma "La Sapienza" \\ P.le A. Moro 2, 00185 Roma, Italy. \\ e-mail: carpi@mat.uniroma1.it
}

\begin{abstract}
We study the problem of recovering Wightman conserved currents from the canonical local implementations of symmetries which can be constructed in the algebraic framework of quantum field theory, in the limit in which the region of localization shrinks to a point. We show that, in a class of models of conformal quantum field theory in space-time dimension $1+1$, which includes the free massless scalar field and the $S U(N)$ chiral current algebras, the energy-momentum tensor can be recovered. Moreover we show that the scaling limit of the canonical local implementation of $S O(2)$ in the free complex scalar field is zero, a manifestation of the fact that, in this last case, the associated Wightman current does not exist.
\end{abstract}

\footnotetext{
${ }^{0}$ Original article published in Reviews in Mathematical Physics 11 (1999), no. 5, 519532
} 


\section{Introduction}

In classical relativistic field theory Noether's theorem associates a conserved current to every one-parameter group of symmetries of the Lagrangian. Moreover the zero component of this current is a density for the infinitesimal generator of this one-parameter group. Although the presence of conserved currents related to symmetries is a general feature of models of quantum field theory, the understanding of this relation in this context is less satisfactory that in the classical case. In the Lagrangian approach to quantum field theory, for example, a classical symmetry can disappear at the quantum level because of the renormalization procedure. Moreover the classical expression of the currents does not give a well defined quantum field because it involves multiplications of the basic fields at the same point, so that, to give a precise definition of the current, one need a further renormalization (for a discussion see [14]). On the other hand, if one starts from general assumptions as the Wightman axioms [16], the existence of such conserved currents must be postulated.

In the algebraic formulation of quantum field theory ("local quantum physics" [10]) a new approach towards a quantum Noether's theorem has been conceived by Doplicher in [5] and developed by Doplicher, Longo and Buchholz in [8] and [2]. In these works it has been proved that, in a theory where the field net satisfies the split property (see [10] and the references quoted there for the meaning and the relevance of the split property in quantum field theory), the global symmetries, including discrete symmetries, space-time symmetries and supersymmetries, can be locally implemented by unitary operators which are canonically constructed from the theory in question. If a part of the symmetries considered forms a connected Lie group, 
then the generators of the corresponding local implementations can be considered as the analogue of the zero component of Wightman conserved currents, smeared with appropriate test functions with support in the region of localization.

It has been suggested by Doplicher in [5] (see also [6] and [7]) that the canonical local generators constructed using the split property could be used to construct Wightman current by an appropriate scaling limit in which the region of localization shrinks to a point. The success of this program would give us a complete quantum Noether's theorem and a general prescription to construct Wightman fields with a definite physical meaning, directly from the algebra of observables.

In this paper we study this problem in some models of chiral field theories, which are a special class of conformal field theories in $1+1$ space time dimensions (see [12] and[4]) that live in the real line, hoping that this will give some enlightenment on the study of a more general situation. The choice of chiral field theories is motivated by their simplicity and by the fact that dilation invariance permits to treat scaling limits in an intrinsic way.

We show that, in a class of models of chiral theories, which includes the free massless scalar field and models arising from representation theory of certain loop groups, the energy-momentum tensor can be recovered in the scaling limit of the canonical local generator of translations constructed by the prescriptions given in [2]. Moreover we show that in the case of the free massless complex field, if we consider the canonical local generator of the $S O(2)$ symmetry, the scaling limit is zero. We interpret this result as a consequence of the fact that the conserved current associated to the phase transformation is not a well defined Wightman field, because of the typical infrared problems of the two-dimensional case. 
One of the key ingredients of this work is the analysis given in [11] for the construction of pointlike localized fields from conformally invariant Haag -Kastler nets.

The present paper is organized in the following way: in section 2 we give assumptions without referring to particular models and show that they are sufficient to recover the energy-momentum tensor (a priori supposed to exist) in the scaling limit of appropriate bounded functions of the canonical local generators. Some of these assumptions are standard but others can be justified only because they work in a non empty class of models, providing us some non trivial examples in which the general program can be realized. Their abstraction from the models is then motivated only by the hope of simplifying the exposition and giving a clear idea of the limits of a possible generalization to models not considered in this paper. In section 3 we show that assumptions of section 2 are satisfied in the models cited above, completing our discussion about the energy-momentum tensor. In section 4 we consider the case of the complex scalar field and finally in section 5 we make some concluding remarks.

\section{General Assumptions, Results and Proofs}

Let $\mathcal{K}$ denote the set of non empty open bounded intervals of the real line $R$. We consider a family $\mathcal{F}=(\mathcal{F}(I))_{I \in \mathcal{K}}$ of von Neumann algebras (the field algebra), acting on a separable Hilbert space $\mathcal{H}$ and we assume that this family satisfies the following properties.

(i) Isotony:

$$
\mathcal{F}\left(I_{1}\right) \subset \mathcal{F}\left(I_{2}\right) \text { for } I_{1} \subset I_{2}, \quad I_{1}, I_{2} \in \mathcal{K} \text {. }
$$


(ii) Locality:

$$
\mathcal{F}\left(I_{1}\right) \subset \mathcal{F}\left(I_{2}\right)^{\prime} \text { for } I_{1} \cap I_{2}=\emptyset, I_{1}, I_{2} \in \mathcal{K}
$$

(iii) There exists a strongly continuous unitary representation $U$ of $S L(2, R)$ in $\mathcal{H}$ such that

$$
U(-1)=1
$$

and

$$
U(\alpha) \mathcal{F}(I) U(\alpha)^{-1}=\mathcal{F}(\alpha I) \text { for } I, \alpha I \in \mathcal{K},
$$

where

$$
S L(2, R) \ni \alpha=\left(\begin{array}{ll}
a & b \\
c & d
\end{array}\right)
$$

acts on $R \bigcup\{\infty\}$ by

$$
x \rightarrow \alpha x=\frac{a x+b}{c x+d}
$$

(note that for every $I \in \mathcal{K}$ we have $\alpha I \in \mathcal{K}$ if $\alpha$ is close enough to 1 ).

(iv) The conformal Hamiltonian $L_{0}$, which generates the restriction of $U$ to the one-parameter group

$$
t \rightarrow\left(\begin{array}{cc}
\cos \frac{t}{2} & -2 \sin \frac{t}{2} \\
\frac{1}{2} \sin \frac{t}{2} & \cos \frac{t}{2}
\end{array}\right)
$$

has non-negative spectrum.

(v) There is a unique (up to a phase) $U$-invariant unit vector $\Omega \in \mathcal{H}$ (the vacuum vector).

(vi) $\mathcal{H}$ is the smallest closed subspace containing $\Omega$ which is invariant for $U$ and $\mathcal{F}(I)$ for every $I \in \mathcal{K}$.

(vii) Split property:

Let $I, \tilde{I} \in \mathcal{K}$ such that the closure of $I$ is contained in $\tilde{I}$ (we write $I \subset \subset \tilde{I}$ ). Then there exists a factor of type I $\mathcal{N}$ such that: 


$$
\mathcal{F}(I) \subset \mathcal{N} \subset \mathcal{F}(\tilde{I}) .
$$

(viii) There exists a strongly continuous unitary representation $V$ of a compact group $G$ leaving the vacuum invariant, commuting with $U$ and such that

$$
V(g) \mathcal{F}(I) V(g)^{-1}=\mathcal{F}(I) \text { for } g \in G \text { and } I \in \mathcal{K} .
$$

(ix) Let $T(a)=U\left(\begin{array}{ll}1 & a \\ 0 & 1\end{array}\right)$ be the group of translations. There exists a Wightman field $\Theta$ (the energy-momentum tensor) given on an invariant dense domain $D_{\Theta}$ containing the vacuum, such that: $\Theta(f)$ is essentially self-adjoint for every function $f \in \mathcal{S}_{R}$ ( $\mathcal{S}_{R}$ is the space of real Schwartz test functions); if $\mathcal{A}(I):=\{F \in \mathcal{F}(I): V(g) F=F V(g)\}$ for $I \in \mathcal{K}$ (the observable algebra) then

$$
e^{i \Theta(f)} \in \mathcal{A}(I),
$$

for $f \in \mathcal{S}_{R}$ with support contained in $I$;

$$
U(\alpha) \Theta(x) U(\alpha)^{-1}=\left(\frac{d \alpha x}{d x}\right)^{2} \Theta(\alpha x) \text { for } \alpha \in S L(2, R) ;
$$

and if $f \in \mathcal{S}_{R}$ is such that $f(x)=1$ for $x \in I$ then

$$
e^{i a \Theta(f)} F e^{-i a \Theta(f)}=T(a) F T(-a) \text { if } F, T(a) F T(-a) \in \mathcal{F}(I) .
$$

(x) Let $\mathcal{H}_{0}$ be the $V$-invariant subspace of $\mathcal{H}$. Because of the positivity of $L_{0}$ the representation $U$ splits into a direct sum of irreducible representations $\tau$ acting on a subspace $\mathcal{H}_{\tau}$ (see [15] and cf. [11]). The equivalence class of each $\tau$ is determined by a non-negative integer $n(\tau)$ which is the lower bound of the spectrum (which is discrete and simple) of the restriction of $L_{0}$ to $\mathcal{H}_{\tau}$. We 
then assume that, in the decomposition of the restriction of $U$ to $\mathcal{H}_{0}$ (which is $U$-invariant because $U$ commute with $V$ ), there appear no representations $\tau$ with $n(\tau)=1$ and only one representation $\tau$ with $n(\tau)=2$.

Note that only here we have a significant restriction for the group $G$ that until now could be taken to be the trivial group.

From the first six assumptions several results can be proved. For example PCT theorem, Haag duality, additivity, the Reeh-Schlieder property (see [11], [13] and [1]). In particular it has been proved in [11] that these assumptions imply the existence of pointlike localized fields naturally associated to $\mathcal{F}$ and the existence of local operator product expansions.

The split property, together with the Reeh-Schlieder property and locality, implies that, if for $I, \tilde{I} \in \mathcal{K}$ we have $I \subset \subset \tilde{I}$, then the triple $\Lambda=(\mathcal{F}(I), \mathcal{F}(\tilde{I}), \Omega)$ is a standard and split inclusion of von Neumann algebras (see [9]). By the results in [2] there exists a local canonical implementation of the translations $T_{\Lambda}(a)=e^{i a P_{\Lambda}}$ in the sense that:

$$
T_{\Lambda}(a) F T_{\Lambda}(-a)=T(a) F T(-a) \text { for } F, T(a) F T(-a) \in \mathcal{F}(I)
$$

and

$$
T_{\Lambda}(a) \in \mathcal{F}(\tilde{I})
$$

Using the transformation properties for the canonical implementations of symmetries (see [2]) we also have

$$
\begin{array}{r}
T_{\Lambda}(a) \in \mathcal{A}(\tilde{I}) \\
T(x) T_{\Lambda}(a) T(-x)=T_{\Lambda+x}(a) \\
D(\lambda) T_{\Lambda}(a) D(\lambda)^{-1}=T_{\lambda \Lambda}(\lambda a)
\end{array}
$$


where $D(\lambda)=U\left(\begin{array}{cc}\lambda^{\frac{1}{2}} & 0 \\ 0 & \lambda^{-\frac{1}{2}}\end{array}\right)$ is the group of dilations and $\Lambda+x, \lambda \Lambda$ are the triples associated to the pairs $I+x, \tilde{I}+x$ and $\lambda I, \lambda \tilde{I}$ respectively.

Heuristically one may think of $P_{\Lambda}$ as an analogue of $\Theta\left(f_{\Lambda}\right)$, where $f_{\Lambda}$ is a real function with the support in $\tilde{I}$ and equal to 1 in $I$ (cf. [6], [7] and [8]). In fact they differ by a perturbation in $\mathcal{F}(I)^{\prime} \cap \mathcal{F}(\tilde{I})$. Then one has the (heuristic) estimate

$$
\int \varphi(x) P_{\lambda \Lambda+x} d x \sim_{\lambda \rightarrow 0} \lambda \int f_{\Lambda}(x) d x \Theta(\varphi) \text { for } \varphi \in \mathcal{S}
$$

( $\mathcal{S}$ is the complex space of Schwartz test functions).

To avoid problems with the domain of $P_{\Lambda}$ (we don't know if it contains the vacuum) we prefer to consider $\frac{i}{\lambda}\left[T_{\lambda \Lambda}(\lambda a)-\left(\Omega, T_{\lambda \Lambda}(\lambda a) \Omega\right)\right]$ instead of $P_{\lambda \Lambda}$ (the vacuum mean value subtraction is a necessary renormalization prescription). Then the previous estimate suggests

$$
\lim _{\lambda \rightarrow 0} \lambda^{-2} \int \varphi(x) T(x) D(\lambda)\left[T_{\Lambda}(a)-\left(\Omega, T_{\Lambda}(a) \Omega\right)\right] D(\lambda)^{-1} T(x)^{-1}=\eta \Theta(\varphi)
$$

where $\eta$ is a constant independent of $\varphi$.

Actually a further regularization is needed to avoid possible singularities of the limit, corresponding to the non integrability of $f_{\Lambda}$ (cf. [11]). Let $\mu$ be the Haar measure on $S L(2, R)$ and $h \in C^{\infty}(S L(2, R))$ have a compact support and integral equal to 1 . For every bounded operator $B$ on $\mathcal{H}$ we consider $B_{h}=\int h(\alpha) U(\alpha) B U(\alpha)^{-1} d \mu(\alpha)$. We can now state the following theorem.

Theorem. For every $h$ as described above with support sufficiently close to the identity there is a constant $\eta$ such that for, every $\varphi \in \mathcal{S}$ with support contained in an open interval $J \in \mathcal{K}$ we have

$$
\lim _{\lambda \rightarrow 0} \lambda^{-2} \int \varphi(x) T(x) D(\lambda)\left[T_{\Lambda}(a)-\left(\Omega, T_{\Lambda}(a) \Omega\right)\right]_{h} D(\lambda)^{-1} T(x)^{-1} d x \psi=
$$




$$
=\eta \Theta(\varphi) \psi
$$

for every $\psi \in \mathcal{F}(J)^{\prime} \Omega$, in the weak topology of $\mathcal{H}$.

Proof. If the support of $h$ is sufficiently close to the identity then

$$
\left[T_{\Lambda}(a)-\left(\Omega, T_{\Lambda}(a) \Omega\right)\right]_{h} \in \mathcal{A}(\hat{I})
$$

for some $\hat{I} \in \mathcal{K}$ so that

$$
\int \varphi(x) T(x) D(\lambda)\left[T_{\Lambda}(a)-\left(\Omega, T_{\Lambda}(a) \Omega\right)\right]_{h} D(\lambda)^{-1} T(x)^{-1} d x \in \mathcal{A}(J)
$$

for $\lambda$ sufficiently small. It is then clear that it is enough to prove our assertion when $\psi=\Omega$.

Let $\mathcal{H}_{\Theta}$ be the closure of the subspace $\{\Theta(f) \Omega: f \in \mathcal{S}\}$. Then, by the covariance of $\Theta$ with respect to $S L(2, R)$, we see that the restriction of $U$ to $\mathcal{H}_{\Theta}$ is irreducible (see [11]). Let $P_{\Theta}$ be the orthogonal projection onto $\mathcal{H}_{\Theta}$. Following the arguments given in [11] in the construction of pointlike localized fields, and using the fact that $\Theta$ can be identified with (a multiple of) the field $\varphi_{\Theta}$ associated to $\mathcal{H}_{\Theta}$ which has been constructed in [11] we find that

$$
\begin{gathered}
\lim _{\lambda \rightarrow 0} \lambda^{-2} P_{\Theta} \int \varphi(x) T(x) D(\lambda)\left[T_{\Lambda}(a)-\left(\Omega, T_{\Lambda}(a) \Omega\right)\right]_{h} D(\lambda)^{-1} T(x)^{-1} d x \Omega= \\
=\eta \Theta(\varphi) \Omega
\end{gathered}
$$

for every $\varphi \in \mathcal{S}$ and an appropriate constant $\eta$. Since, by assumption (x), $\mathcal{H}_{\Theta}$ is the only (closed) subspace of $\mathcal{H}_{0}$ which is irreducible for $U$ with conformal dimension equal to one or equal to two, for every orthogonal projection $P_{\tau}$ onto a $U$-irreducible subspace $\mathcal{H}_{\tau}$ orthogonal to $\mathcal{H}_{\Theta}$ we have, following again the arguments given in [11],

$$
\lim _{\lambda \rightarrow 0} \lambda^{-2} P_{\tau} \int \varphi(x) T(x) D(\lambda)\left[T_{\Lambda}(a)-\left(\Omega, T_{\Lambda}(a) \Omega\right)\right]_{h} D(\lambda)^{-1} T(x)^{-1} d x \Omega=
$$


for every $\varphi \in \mathcal{S}$. Then if $\psi$ is in the linear span of finitely many $U$ - irreducible subspaces of $\mathcal{H}$ we have

$$
\begin{aligned}
\lim _{\lambda \rightarrow 0} \lambda^{-2}\left(\psi, \int \varphi(x) T(x) D(\lambda)\right. & {\left.\left[T_{\Lambda}(a)-\left(\Omega, T_{\Lambda}(a) \Omega\right)\right]_{h} D(\lambda)^{-1} T(x)^{-1} d x \Omega\right)=} \\
= & (\psi, \eta \Theta(\varphi) \Omega) .
\end{aligned}
$$

Since the set of such vectors $\psi$ is dense in $\mathcal{H}$, to prove the weak convergence it is enough to show that the norm of

$$
\lambda^{-2} \int \varphi(x) T(x) D(\lambda)\left[T_{\Lambda}(a)-\left(\Omega, T_{\Lambda}(a) \Omega\right)\right]_{h} D(\lambda)^{-1} T(x)^{-1} d x \Omega
$$

is bounded with respect to $\lambda$.

We have

$$
\begin{aligned}
& \left\|\lambda^{-2} \int \varphi(x) T(x) D(\lambda)\left[T_{\Lambda}(a)-\left(\Omega, T_{\Lambda}(a) \Omega\right)\right]_{h} D(\lambda)^{-1} T(x)^{-1} d x \Omega\right\|^{2}= \\
& \quad=\lambda^{-4} \iint \bar{\varphi}(y) \varphi(x) \\
& \left(\Omega,\left[T_{\Lambda}(a)-\left(\Omega, T_{\Lambda}(a) \Omega\right)\right]_{h}^{*} T\left(\frac{x-y}{\lambda}\right)\left[T_{\Lambda}(a)-\left(\Omega, T_{\Lambda}(a) \Omega\right)\right]_{h} \Omega\right) d y d x .
\end{aligned}
$$

By the conformal cluster theorem [11] the Fourier transform of

$$
\left(\Omega,\left[T_{\Lambda}(a)-\left(\Omega, T_{\Lambda}(a) \Omega\right)\right]_{h}^{*} T(x)\left[T_{\Lambda}(a)-\left(\Omega, T_{\Lambda}(a) \Omega\right)\right]_{h} \Omega\right)
$$

can be written as $\vartheta(p) p^{3} F(p)$, where $\vartheta(p)$ is the Heaviside step function and $F(p)$ is an analytic function of rapid decrease (see [11]) so that we have

$$
\begin{gathered}
\left\|\lambda^{-2} \int \varphi(x) T(x) D(\lambda)\left[T_{\Lambda}(a)-\left(\Omega, T_{\Lambda}(a) \Omega\right)\right]_{h} D(\lambda)^{-1} T(x)^{-1} d x \Omega\right\|^{2}= \\
=\int_{0}^{\infty}|\hat{\varphi}(p)|^{2} F(\lambda p) p^{3} d p \leq \max F \int_{0}^{\infty}|\hat{\varphi}(p)|^{2} p^{3} d p
\end{gathered}
$$


Q.E.D.

We observe now that this theorem gives a positive answer to our problem if we can show that the constant $\eta$ is not zero. From the non-vanishing argument given in [11] it follows that if

$$
P_{\Theta} T_{\Lambda}(a) \Omega \neq 0
$$

an accidental vanishing of $\eta$ for a given function $h$ can be avoided by an arbitrarily small translation of $h$ on $S L(2, R)$. Unfortunately we are not able to prove the above condition. However we avoid this problem with the aid of the following proposition.

Proposition. There exist values of $x$ and $a$, for arbitrarily large $|x|$, such that

$$
P_{\Theta} T_{\Lambda}(a) T_{\Lambda+x}(a) \Omega \neq 0
$$

Proof. Let's suppose the contrary, i.e.

$$
P_{\Theta} T_{\Lambda}(a) T_{\Lambda+x}(a) \Omega=0
$$

for every $a$ and $x$ such that $|x|>L$. Without loss of generality we can assume $L$ greater than the diameter of the interval $\tilde{I}$ where $T_{\Lambda}$ is localized. For every $x$ such that $|x|>L$, we can choose a function $f_{x} \in \mathcal{S}_{R}$ such that, for $\epsilon$ small enough

$$
e^{i \epsilon \Theta\left(f_{x}\right)} T_{\Lambda}(a) T_{\Lambda+x}(a) e^{-i \epsilon \Theta\left(f_{x}\right)}=T_{\Lambda}(a) T_{\Lambda+\epsilon+x}(a) .
$$

It follows that

$$
\begin{gathered}
\frac{d}{d x}\left(\Omega, T_{\Lambda}(a) T(x) T_{\Lambda}(a) \Omega\right)= \\
=i\left(\Theta\left(f_{x}\right) \Omega, T_{\Lambda}(a) T_{\Lambda+x}(a) \Omega\right)-i\left(\Omega, T_{\Lambda}(a) T_{\Lambda+x}(a) \Theta\left(f_{x}\right) \Omega\right)=
\end{gathered}
$$


for $|x|>L$ and then (using the conformal cluster theorem) that the function

$$
x \rightarrow\left(\Omega, T_{\Lambda}(a) T(x) T_{\Lambda}(a) \Omega\right)-\left(\Omega, T_{\Lambda}(a) \Omega\right)^{2}
$$

has compact support. Then, using the positivity of the generator of $T$ (positivity of the energy) we have

$$
\left(\Omega, T_{\Lambda}(a) T(x) T_{\Lambda}(a) \Omega\right)=\left(\Omega, T_{\Lambda}(a) \Omega\right)^{2}
$$

for every $a, x \in R$. In particular we have

$$
\left(\Omega, T_{\Lambda}(2 a) \Omega\right)=\left(\Omega, T_{\Lambda}(a) \Omega\right)^{2} .
$$

Let $E_{0}$ be the orthogonal projection onto the subspace of $\mathcal{H}$ spanned by $\Omega$. Using the conformal cluster theorem it is not difficult to prove that in the limit $a \rightarrow \infty T(a)$ converges to $E_{0}$ in the weak topology of $B(\mathcal{H})$ (cf. [16]). Thus $T(a) \otimes 1$ converges to $E_{0} \otimes 1$ in the weak topology of $B(\mathcal{H} \otimes \mathcal{H})$. Since $E_{0} \otimes 1$ is the orthogonal projection onto the $T \otimes 1$-invariant subspace of $\mathcal{H} \otimes \mathcal{H}$ and since $T_{\Lambda}$ is unitarily equivalent to $T \otimes 1$ [8], the previous equation leads to

$$
\left(\Omega, E_{\Lambda} \Omega\right)=\left(\Omega, E_{\Lambda} \Omega\right)^{2}
$$

where $E_{\Lambda}$ is the orthogonal projection onto the $T_{\Lambda^{-}}$invariant subspace of $\mathcal{H}$. Since $\Omega$ cannot be $T_{\Lambda}$-invariant because of the Reeh-Schlieder property, the previous equality implies that

$$
\left(\Omega, E_{\Lambda} \Omega\right)=0,
$$

so that, using the fact that $E_{\Lambda}$ is local and different from 0 , we are led the desired contradiction. 
It is now clear that if we use $T_{\Lambda}(a) T_{\Lambda+x}(a)$, which implements the translations in a disconnected region (here we take $x$ large), instead of $T_{\Lambda}(a)$, then the result of the previous theorem can be strengthened by the fact that the constant $\eta$ is different from zero for a suitable choice of $h, a$ and $x$.

Remark. We have proved that the scaling limit converges on the dense domain $\mathcal{F}(J)^{\prime} \Omega$ when the test function $\varphi$ has support in the open interval $J$. We will show in the appendix that, for every $J \in K, \mathcal{F}(J)^{\prime} \Omega$ contains a core for $L_{0}$. Thus, in typical models, the energy-bounds proved in [3] imply that $\mathcal{F}(J)^{\prime} \Omega$ is a core for $\Theta(\varphi)$.

\section{The Models}

In this section we consider some models satisfying the assumptions of section 2. Since these models are standard, here we sketch most of their properties referring to the literature for the details (in particular to [12], [4] and [13]).

\section{The Free Hermitian Scalar Field}

This theory is generated by a hermitian Wightman field $j$ on the real line (the $U(1)$-current ) satisfying the canonical commutation relations

$$
[j(x), j(y)]=i \delta^{\prime}(x-y)
$$

We can define the local field algebras by

$$
\mathcal{F}(I)=\left\{j(f): f \in \mathcal{S}_{R} \text { and } \operatorname{supp} f \subset I\right\}^{\prime \prime} \text { for } I \in \mathcal{K} .
$$

By the results in [3] and [13] it follows that assumptions from (i) to (vi) of section 2 are satisfied (see also [12] and [4]). The transformation $j \rightarrow-j$ leaves all the Wightman functions invariant and so they can be unitarily implemented (see [16]) giving a representation of $Z_{2}$ which satisfies assumption 
(viii). An energy-momentum tensor satisfying assumption (ix) is given by

$$
\Theta(x)=\frac{1}{2}: j^{2}:(x),
$$

where the double dots indicate the Wick product. Assumption (x) can be proved by showing that in $\mathcal{H}_{0}$ there is only one (up to a multiplicative constant) eigenvector of $L_{0}$ corresponding to the eigenvalue 2 and no eigenvectors corresponding to the eigenvalue 1 (here we are using the properties of the irreducible representations with positive conformal Hamiltonian stated above). To prove the last statement we use the fact that for every positive integer $n$ the corresponding eigenspace is given by the linear span of vectors of the following form

$$
J_{-n_{1}} J_{-n_{2}} \ldots J_{-n_{k}} \Omega
$$

with

$$
\begin{aligned}
& n_{1} \geq n_{2} \ldots \geq n_{k} \geq 1, \\
& n_{1}+n_{2} \ldots+n_{k}=n,
\end{aligned}
$$

where for every integer $m, J_{m}$ is the $m$-th Fourier component of $j$ (see [4] ). Since for every integer $m$ the projection of $J_{m} \Omega$ onto $\mathcal{H}_{0}$ is zero (because $V(-1) J_{m} V(-1)=-J_{m}$ ) then the only eigenvector (up to a multiplicative constant) of $L_{0}$ in $\mathcal{H}_{0}$ corresponding to the eigenvalue 2 is given by

$$
J_{-1} J_{-1} \Omega
$$

Finally the split property (assumption (vii)) follows from the finiteness of the trace of $e^{-\beta L_{0}}$ for positive $\beta$ together with an appropriate estimate for $\beta \rightarrow 0[13]$.

Chiral current algebras of simple Lie groups

Let $G$ a connected, simply connected, simple, simply laced and compact 
Lie group and let $L G$ be its Lie algebra. We consider theories arising from vacuum representations of the Kac-Moody algebra associated to $G$ (see [12] and [4]). They are generated by a family of hermitian Wightman currents $\left\{j^{u}: u \in L G\right\}$ such that the map $u \rightarrow j^{u}$ is $R$-linear and, for every $u, v \in L G$,

$$
\left[j^{u}(x), j^{v}(y)\right]=i j^{[u, v]}(x) \delta(x-y)+i k<u, v>\delta^{\prime}(x-y),
$$

where $k$ is a positive constant and $\langle\cdot, \cdot\rangle$ is the unique (up to a normalization) invariant scalar product on $L G$. With an appropriate normalization for $<\cdot, \cdot>$, the possible values of $k$ (levels) are restricted to be positive integers. If we define the field net by

$$
\mathcal{F}(I)=\left\{j^{u}(f): u \in L G, f \in \mathcal{S}_{R}, \operatorname{supp} f \subset I\right\}^{\prime \prime} \text { for } I \in \mathcal{K}
$$

then, by the results in [3] and [13], all the assumptions from (i) to (vii) are satisfied and there is a representation $V$ of $G$ with the properties of (viii) and such that, for every $u \in L G, g \in G$

$$
V(g) j^{u} V(g)^{-1}=j^{g u}
$$

(with $g u$ we denote the adjoint representation of $G$ on $L G$ ). An energymomentum tensor $\Theta$ with the properties of (ix) is obtained by the Sugawara construction (see [12], [4] and [13]). Finally we show the validity of assumption $(\mathrm{x})$.

For every positive integer $n$ the corresponding eigenspace of $L_{0}$ is given by the linear span of vectors of the following form

$$
J_{-n_{1}}^{u_{1}} J_{-n_{2}}^{u_{2}} \ldots J_{-n_{k}}^{u_{k}} \Omega
$$

with

$$
n_{1} \geq n_{2} \ldots \geq n_{k} \geq 1
$$




$$
n_{1}+n_{2} \ldots+n_{k}=n,
$$

where $u_{1}, \ldots, u_{k} \in L G$ and, for every integer $m$ and $u \in L G, J_{m}^{u}$ is the $m$-th Fourier component of $j^{u}$ (see [4]). If $P_{0}$ is the orthogonal projection onto $\mathcal{H}_{0}$ then we have, for every integer $m$ and $u \in L G$

$$
P_{0} J_{m}^{u} \Omega=0
$$

Moreover there exists a vector $\psi \in \mathcal{H}$ such that, for every $u, v \in L G$

$$
P_{0} J_{-1}^{u} J_{-1}^{v} \Omega=<u, v>\psi
$$

By the same argument given above, this implies that assumption (x) holds.

Before concluding this section, we shortly describe the case of the chiral current algebras of some semisimple compact Lie groups. We consider a group $G$ which is the direct product of a finite number $N$ of connected, simply connected, simply laced, simple compact Lie groups $G_{i} ; i=1, \ldots, N$. The corresponding local field algebras are given, for every $I \in \mathcal{K}$, by

$$
\mathcal{F}(I)=\mathcal{F}_{1}(I) \otimes \ldots \otimes \mathcal{F}_{N}(I),
$$

where, for $i=1, \ldots, N, \mathcal{F}_{i}$ is the field net generated by the chiral current algebra of $G_{i}$ considered above. Moreover the vacuum representation of $\mathcal{F}$ is the tensor product of the vacuum representations of $\mathcal{F}_{i} ; i=1, \ldots, N$. The energy-momentum tensor is given by

$$
\Theta(x)=\sum_{i=1}^{N} \Theta_{i}(x)
$$

where $\Theta_{i}$ is the Sugawara energy-momentum tensor corresponding to $G_{i}$. Besides it is not difficult to show that, for every triple $\Lambda$, the canonical local implementation of the translations is given by

$$
T_{\Lambda}=T_{\Lambda}^{1} \otimes \ldots \otimes T_{\Lambda}^{N}
$$


Thus, a similar analysis to that given in section 2, shows that, for every infinitely differentiable function $h$ on $S L(2, R)$ with support close enough to the identity and for every $\varphi \in \mathcal{S}$ such that $\operatorname{supp} \varphi \in J(J \in \mathcal{K}$ open), we have

$$
\begin{gathered}
\lim _{\lambda \rightarrow 0} \lambda^{-2} \int \varphi(x) \\
T(x) D(\lambda)\left[T_{\Lambda}(a) T_{\Lambda+y}(a)-\left(\Omega, T_{\Lambda}(a) T_{\Lambda+y}(a) \Omega\right)\right]_{h} D(\lambda)^{-1} T(x)^{-1} d x \psi= \\
=\sum_{i=1}^{N} \eta_{i} \Theta_{i}(\varphi) \psi
\end{gathered}
$$

for every $\psi \in \mathcal{F}(J)^{\prime} \Omega$, in the weak topology of $\mathcal{H}$. Moreover for every $i$ an accidental vanishing of $\eta_{i}$ can be avoided by an appropriate choice of $y$ and $a$ and by an arbitrarily small translation of $h$. Unfortunately we are not able to prove in general that all the constants $\eta_{i}$ must be equal. So, in this case the result is less satisfactory than that given for a simple group. However, in the particular case in which all the groups $G_{i}$ are equal, the equality of the constants $\eta_{i}$ follows from the symmetry under the permutation group $S_{N}$.

\section{A "Bad" Example}

In this section we consider the free complex scalar field. This model is generated by two commuting chiral currents $j_{1}$ and $j_{2}$. For the two point Wightman functions we have

$$
\begin{array}{r}
\left(\Omega, j_{1}(x) j_{2}(y) \Omega\right)=\left(\Omega, j_{2}(x) j_{1}(y) \Omega\right)=0 \\
\left(\Omega, j_{1}(x) j_{1}(y) \Omega\right)=\left(\Omega, j_{2}(x) j_{2}(y) \Omega\right) .
\end{array}
$$

The transformations

$$
\begin{aligned}
j_{1}(x) & \rightarrow \cos \vartheta j_{1}(x)+\sin \vartheta j_{2}(x), \\
j_{2}(x) & \rightarrow \cos \vartheta j_{2}(x)-\sin \vartheta j_{1}(x)
\end{aligned}
$$


leave then all the Wightman functions invariant and so can be implemented by a unitary representation $S$ of $S O(2)$ leaving the vacuum invariant. Since this model is the tensor product of two free scalar Hermitian field models, the split property is fulfilled. So, by the results in [8], we can consider the canonical local implementations $S_{\Lambda}$ of $S$. In this case, as we are considering a gauge symmetry (corresponding to a dimensionless charge), the correct scaling factor in the scaling limit is $\lambda^{-1}$. Let $\mathcal{H}_{0}$ be the $S$ - invariant subspace of $\mathcal{H}$. An argument similar to those given in the previous section shows that, in $\mathcal{H}_{0}$, there are no irreducible components of the representation $U$ of $S L(2, R)$ with conformal dimension equal to one. Moreover, by the transformation properties of $S_{\Lambda}[2]$ we have

$$
S(\vartheta) S_{\Lambda}\left(\vartheta^{\prime}\right) S(\vartheta)^{-1}=S_{\Lambda}\left(\vartheta^{\prime}\right)
$$

so by using the results in [11] we get the following proposition.

Proposition: Let $h$ an infinitely differentiable function on $S L(2, R)$ with compact support. For every $\varphi \in \mathcal{S}$ with the support contained in some open interval $J \in \mathcal{K}$ we have

$$
\lim _{\lambda \rightarrow 0} \lambda^{-1} \int \varphi(x) T(x) D(\lambda)\left[S_{\Lambda}(\vartheta)-\left(\Omega, S_{\Lambda}(\vartheta) \Omega\right)\right]_{h} D(\lambda)^{-1} T(x)^{-1} \psi=0,
$$

for every $\psi \in \mathcal{F}(J)^{\prime} \Omega$, in the weak topology of $\mathcal{H}$.

This result cannot be avoided using the techniques explained in section 2 . Thus we cannot obtain a Wightman current corresponding to the symmetry $S$ in the scaling limit of the canonical local implementations. This fact however is not surprising because in this case this Wightman current does not exist. In fact the classical expression for this current given by the Noether's theorem does not define a Wightman field because of the infrared divergences typical 
of the $1+1$ dimensional models (by the same reason a proper scalar Wightman field does not exist, cf. [14]).

\section{Conclusions}

We have shown that in some models of conformal field theory the program of recovering Wightman conserved currents from the local canonical implementations of symmetries has a positive issue. We can summarize the ingredients for this success in the following two points:

(1) the existence of a local operator product expansion;

(2) the transformation properties of the canonical local implementations of symmetries.

Although these are general features of models of quantum field theory, we believe that they will not be sufficient, even if necessary, in the study of a more general situation including field theories in a four dimensional spacetime. In this study the property of local implementation, which is never used directly in the present work, should play a prominent part. A direct use of this property should also permit to give positive results without assuming the existence of the Wightman currents. In this way we should obtain a prescription for their construction.

Acknowledgements. Special thanks are due to Prof. S. Doplicher for having suggested the problem, enlightening discussions and a constant encouragement. We also wish to thank Prof. K. Fredenhagen for some very useful discussions and hints. 


\section{Appendix}

In this appendix we show that, for every open interval $I \in \mathcal{K}$, the domain $\mathcal{F}(I) \Omega$ contains a core for $L_{0}$. This fact is an easy corollary of the following lemma.

Lemma. Let $\mathcal{B} \subset \mathcal{R}$ be an inclusion of von Neumann algebras given on a separable Hilbert space $\mathcal{H}$ and let $\xi \in \mathcal{H}$ be a cyclic vector for $\mathcal{B}$. Let $U$ be a strongly continuous one-parameter group of unitary operators acting on $\mathcal{H}$ leaving $\xi$ invariant and let $H$ be its self-adjoint generator. If there exists a real number $\delta>0$ such that $U(t) \mathcal{B} U(t)^{-1} \subset \mathcal{R}$ for $|t|<\delta$ then $\mathcal{R} \xi$ contains a core for $H$.

Proof. If $\varphi \in \mathcal{S}$, we denote $\int \varphi(t) U(t) d t$ by $U(\varphi)$. Then our assumptions imply that if $\operatorname{supp} \varphi \subset(-\delta, \delta)$ then

$$
U(\varphi) \mathcal{B} \xi \subset \mathcal{R} \xi
$$

We now show that the domain $\mathcal{D}:=\{U(\varphi) \mathcal{B} \xi: \operatorname{supp} \varphi \subset(-\delta, \delta)\}$ is a core for $H$. If $\psi \in \mathcal{H}$, then $U(\varphi) \psi$ is in the domain of $H$ and

$$
H U(\varphi) \psi=i U\left(\varphi^{\prime}\right) \psi
$$

Since $\xi$ is cyclic for $\mathcal{B}$, for every $\psi \in \mathcal{H}$ we can find a sequence $B_{n} \in \mathcal{B}$ such that $\lim _{n \rightarrow \infty} B_{n} \xi=\psi$ and thus

$$
\begin{aligned}
\lim _{n \rightarrow \infty} U(\varphi) B_{n} \xi & =U(\varphi) \psi \\
\lim _{n \rightarrow \infty} H U(\varphi) B_{n} \xi & =H U(\varphi) \psi .
\end{aligned}
$$

Finally let $\varphi \in \mathcal{S}$ be a positive function with integral equal to one and support contained in $(-\delta, \delta)$ and let $\varphi_{n}$ be defined by $\varphi_{n}(t)=n \varphi(n t)$. Now, 
for every $\psi$ in the domain of $H$ we have

$$
\begin{array}{r}
\lim _{n \rightarrow \infty} U\left(\varphi_{n}\right) \psi=\psi \\
\lim _{n \rightarrow \infty} H U\left(\varphi_{n}\right) \psi=H \psi .
\end{array}
$$

Thus the closure of the graph of the restriction of $H$ to $\mathcal{D}$ contains the graph of $H$ i.e. $\mathcal{D}$ is a core for $H$. Q.E.D.

We take now an open non-empty interval $J \subset \subset I$. Our previous assertion follows from the lemma taking $\mathcal{B}=\mathcal{F}(J), \mathcal{R}=\mathcal{F}(I), \xi=\Omega$ and $H=L_{0}$.

\section{References}

[1] Brunetti R., Guido D., Longo R.: Modular structure and duality in conformal quantum field theory. Commun. Math. Phys. 156, 201 (1993).

[2] Buchholz D., Doplicher S.,Longo R.: On Noether's theorem in quantum field theory. Ann. Phys. 170, 1 (1986).

[3] Buchholz D., Schulz-Mirbach H.: Haag duality in conformal quantum field theory. Rev. Math. Phys. 2, 105 (1990).

[4] Di Francesco Ph., Mathieu P., Sénéchal D.: Conformal field theory. Springer-Verlag, 1996.

[5] Doplicher S.: Local aspects of superselection rules. Commun. Math. Phys. 85, 73 (1982).

[6] Doplicher S.: Current algebra and the nature of symmetries in local quantum field theory. In S. Albeverio and Ph. Blanchard (eds.): Trend and developments in the eighties. World Scientific, 1985. 
[7] Doplicher S.: Local observables and the structure of quantum field theory. In D. Kastler (ed.): Algebraic theory of superselection sectors. World Scientific, 1990.

[8] Doplicher S., Longo R.: Local aspects of superselection rules II. Commun. Math. Phys. 88, 399 (1983).

[9] Doplicher S., Longo R.: Standard and split inclusions of von Neumann algebras. Invent. Math. 75, 493 (1984).

[10] Haag R.: Local Quantum Physics. Springer-Verlag, 1992.

[11] Fredenhagen K., Jörß M.: Conformal Haag-Kastler nets, pointlike localized fields and the existence of operator product expansions. Commun. Math. Phys. 176, 541 (1996).

[12] Furlan P., Sotkov G. M., Todorov I. T.: Two-dimensional conformal field theory. Rivista del Nuovo Cimento 12 (6), 1 (1988).

[13] Gabbiani F., Fröhlich J.: Operator algebras and conformal field theory. Commun. Math. Phys. 155, 569 (1993).

[14] Itzykson C., Zuber J. B.: Quantum field theory. MacGraw Hill, 1980.

[15] Lang S.: $S L_{2}(R)$. Springer-Verlag, 1975.

[16] Streater R. F., Wightman A. S.: PCT spin statistics and all that. Benjamin, 1964. 